\title{
Artificial Intelligence in Cardiac Arrhythmia Classification
}

\author{
Siteng Chen*, Ao Li and Janet Meiling Roveda \\ Electrical and Computer Engineering, USA \\ *Corresponding author: Siteng Chen, Electrical and Computer Engineering, Tucson, AZ, USA

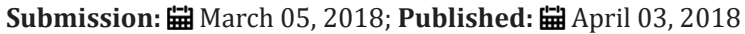

\begin{abstract}
Arrhythmia classification with high precision is usually performed by cardiologists with high time consumption. Automatic arrhythmia classifiers based on artificial intelligence algorithm can help cardiologists to obtain better precision and reduce time consuming. In this mini-review, we compare optimization methods, machine learning methods and deep learning methods in cardiac arrhythmia classification. A high-performance classifier based on deep learning algorithm would be a viable direction of the future research.
\end{abstract}

Keywords: Cardia arrhythmia classification; Artificial intelligence; Machine learning; Deep learning; Convolutional neural network

\section{Introduction}

Cardiac arrhythmia is one of the most important indicators of heart disease. As a common but poorly understood arrhythmia, non-sustained ventricular tachycardia (NSVT) has been recorded in a wide range of conditions, from apparently healthy individuals to patients with significant heart disease [1]. The early prognosis of NSVT is becoming a necessary step in heart failure research, as it can facilitate the subsequent clinical management of patients [2]. In the current medical routines, cardiologists' expertise is required for the diagnosis of life threatening cardiac arrhythmias. The time consumption is a big challenge. Automatic arrhythmia classifier can help cardiologists reduce the time from hours to milliseconds. Although several automatic arrhythmia classification methods based on artificial intelligence have been proposed, these approaches cannot provide end-to-end process. And thus, they still require a good number of cardiologists' hand-on interferences. With the recent state-of-the-art performances obtained by deep learning methods $[3,4]$, it is very encouraging for researchers to implement these techniques to the field of cardiac arrhythmia classification. In this mini-review, we introduce an automatic classification of cardiac arrhythmias that can both provide objective diagnostic results and save time for the cardiologists. We focus on the deep learning based artificial intelligence and its application in the cardiac arrhythmia. We compare existing artificial intelligence methods and applications in cardiac arrhythmia classification to find a viable direction of future research.

\section{Artificial Intelligence in Cardiac Arrhythmia Classification}

A careful investigation of the existing artificial intelligence methods in cardiac arrhythmia reveals three major types:

\section{Optimization methods (OP) based}

The most successful optimization method used in cardiac arrhythmia classification is genetic programming, often paraphrased as "survival of the fittest" [5]. There is a population of individuals that reproduce with each other. Over time, the individuals satisfied most conditions will survive and eventually evolve to do well in the given environment. The capital advantage of genetic programming is that we do not need to worry much about explicit definitions. In Tavassoli et al. [6], fifteen features are manually extracted from Electrocardiography (ECG) signal and seven different types of arrhythmia classes are categorized with an accuracy of $98.75 \%, 98.93 \%, 99.10 \%, 99.46 \%, 99.82 \%, 99.46 \%$ and $99.82 \%$ respectively.

\section{Traditional machine learning methods (ML)}

Support Vector Machine (SVM) is the most common traditional machine learning method was used to do cardiac arrhythmia classification. SVM provides a good performance when selecting input features and tuning parameter properly. In paper [7], one free parameter window size and nine features coming from ECG signals are used to train a two-class classifier, which obtain an accuracy of $98.0 \%$. Besides, other machine learning methods with limit learning capacity, such as shallow Artificial Neural Network (ANN), are used in arrhythmia classification as well.

\section{Deep learning methods (DL)}

The above-mentioned methods are traditional approaches because carefully selected and filtered features are necessary during the training process. On the other hand, DL methods provide end-to-end learning capacity and thus can be applied to a 
wide range of applications. One such DL method is convolutional neural networks (CNNs). Specifically, in DL, learning models can be trained by raw data instead of features labeled by human [1]. In a recent deep learning paper [4], researchers built a 34-layers CNN to identify fourteen types of cardiac arrhythmia. An aggregated accuracy of $80 \%$ is obtained by this deep learning model.

Table 1 shows the summarized comparison of methods from different AI types together with performance. The input format of each classifier, located in the forth column, indicates that optimization method and traditional machine learning method are trained by manually selected features. However deep learning method can handle ECG raw data directly. In the fifth column, arrhythmia type shows classification capability of each method. The DL method shows its advantages in terms of higher capability and including more complex types of arrhythmia.Lower accuracies from deep learning method and higher accuracies from others are listed in the last column. Figure 1 demonstrates the classification capability from 9 different methods [4,6,7-13]. The size of each circle corresponding to the capability of each classifier, which is indicated by the number in circle as well. The blue, green and yellow color represent optimization method, machine learning method and deep learning method respectively. The largest circle with yellow indicates that the deep learning method [7] has maximum classification capability. The above analysis shows that the lack of research on NSVT distinguishing ability. To better predict the underlying he

\section{Conclusion}

The above analysis shows that the lack of research on NSVT distinguishing ability. To better predict the underlying heart disease, NSVT should be included in future studies.

Further, several researchers have addressed the arrhythmia classification problem using raw data or features extracted from ECG signal. Although the performance of deep learning model does not surpass the traditional AI methods, it exhibits the following advantages:
a. Learning from raw data directly.
b. More classes can be identified.
c. No strong cardiology experience is needed.

In conclusion, DL model has proven its capability in cardiac arrhythmia classification. A high-performance deep learning classifier should be developed to solve more complex problems.

\section{References}

1. Isin Ali, Selen Ozdalili (2017) Cardiac arrhythmia detection using deep learning. Procedia Computer Science 120: 268-275.

2. Marine JE (2016) Non sustained ventricular tachycardia in the normal heart. Card Electrophysiol Clin 8(3): 525-543.

3. Krizhevsky A, Ilya S, Geoffrey EH (2017) Image net classification with deep convolutional neural networks. Communications of the ACM 60(6): 84-90.

4. Rajpurkar P, Awni YH, Masoumeh H, Codie B, Andrew YN (2017) Cardiologist level arrhythmia detection with convolutional neural networks.

5. Yasin K, Huseyin P (2015) Feature selection using genetic algorithms for premature ventricular contraction classification. $20159^{\text {th }}$ International Conference on Electrical and Electronics Engineering (ELECO).

6. Tavassoli M, Ebadzadeh M, Malek H (2012) Classification of cardiac arrhythmia with respect to ECG and HRV signal by genetic programming. Machine Learning and Pattern Recognition. 3(1): 1-8.

7. Qiao Li, Cadathur R, Gari DC (2014) Ventricular fibrillation and tachycardia classification using a machine learning approach. IEEE Transactions on Biomedical Engineering 61(6): 1607-1613.

8. Li H, Yuan D, Ma X, Cui D, Cao L (2017) Genetic algorithm for the optimization of features and neural networks in ECG signals classification. Sci Rep 7: 41011.

9. Jalal AN, Mostafa S, Sadoghi HY, Mahmoud N, Bahram N (2009) Intelligent arrhythmia detection using genetic algorithm and Emphatic SVM (ESVM). Third UKSim European Symposium on Computer Modeling and Simulation.

10. Asl BM, Setarehdan SK, Mohebbi M (2008) Support vector machinebased arrhythmia classification using reduced features of heart rate variability signal. Artif Intell Med 44(3): 51-64.

11. Ahmad Arbab M, Gul Muhammad K, Sahibzada Ali M (2013) Classification of arrhythmia types using cartesian genetic programming evolved artificial neural networks. Engineering Applications of Neural Networks Communications in Computer and Information Science. pp. 282-291.

12. AS Al Fahoum, I Howitt (1999) Combined wavelet transformation and radial basis neural networks for classifying life-threatening cardiac arrhythmias. Medical and Biological Engineering and Computing 37(5): 566-573.

13. Song MH, Lee J, Cho SP, Lee KJ, Yoo SK (2005) Support vector machine based arrhythmia classification using reduced features. Int J Control Automat Syst 3(4): 571-579.

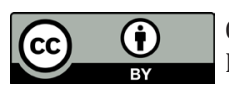

Creative Commons Attribution 4.0 International License

For possible submissions Click Here

\section{Submit Article}

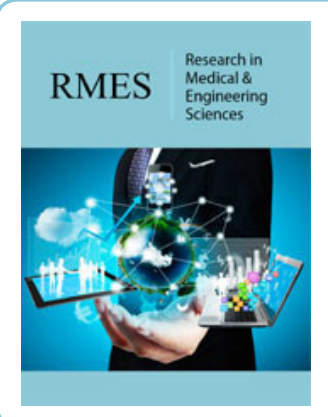

\section{Research in Medical \& Engineering Sciences}

\section{Benefits of Publishing with us}

- High-level peer review and editorial services

- Freely accessible online immediately upon publication

- Authors retain the copyright to their work

- Licensing it under a Creative Commons license

- Visibility through different online platforms 